I M M - NYU 314

NOVEMBER 1963

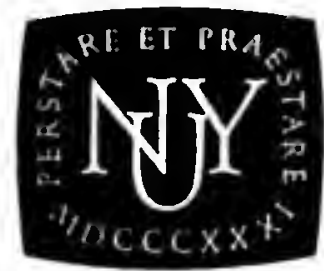

NEW YORK UNIVERSITY

COURANT INSTITUTE OF

MATHEMATICAL SCIENCES

\title{
Asymptotic Efficiency of a Class of c-Sample Tests
}

\author{
MADAN LAL PURI
}

PREPARED UNDER

CONTRACT NONR-285(38)

WITH THE

OFFICE OF NAVAL RESEARCH 
$$
\text { . }
$$ 
NEW YORK UNIVERSITY

Courant Institute of Mathematical Sciences

ASYMPTOTIC EFFICIENCY OF A CLASS OF C-SAMPLE TESTS ${ }^{1}$ Madan Lal Puri

${ }^{1}$ This paper was prepared with the partial support of the Office of Naval Research, Contract Nonr-222-(43), while the author was at the University of California, Berkeley. It was revised at the Courant Institute of Mathematical Sciences, New York University under the sponsorship of the Office of Naval Research, Contract Nonr..285(38). Reproduction in whole or in part is permitted for any purpose of the United States Government. 

I Summarl. For testing the equality of $c$ continuous probability distributions on the basis of $c$ independent random samples, the test statistics of the form

$$
\mathcal{L}=\sum_{j=1}^{c} m_{j}\left[\left(T_{N, j}-\mu_{N, j}\right) / A_{N}\right]^{2}
$$

are considered. Here $m_{j}$ is the size of the jth sample, $\mu_{N, j}$ and $A_{N}$ are normalizing constants, and

$$
T_{N, j}=\frac{I}{m_{j}} \sum_{i=1}^{N} E_{N, i} Z_{N, i}^{(j)}
$$

where $z_{N, i}^{(j)}=1$, if the ith smallest of $N=\frac{N}{i} m_{j}$ observations is from the $j$ th sample and $z_{N, i}^{(j)}=0$ otherwise. Sufficient conditions are given for the joint asymptotic normality of $\mathrm{T}_{N, j} ; j=1, \ldots, c$. Under suitable regularity conditions and the assumption that the ith distribution function is $F\left(x+\theta_{i} / \sqrt{N}\right)$, the limiting distribution of $\mathcal{L}$ is derlved. Finally, the asymptotic relative efficiencles in P1tman's sense of the $\mathcal{L}$ test relative to some of its competitors viz, the Kruskal-Wallis $H$ test (which is a particular case of the $\mathcal{L}$ test) and the classical 7 test are obtained and shown to be independent of the number $c$ of samples. 
$\cdots$

1

$\therefore \quad$ 
2. Introduction. Cne of the frequentily encountered problems in statistics is to decide whether differences in various samples should be rejarded as due to differences in the parent populations or due to chance variations which are to be expected among random samples from the same population. A few tests of nonparametric nature have been proposed for this c-sample problem. The Kruskal-ballis $H$ test [14], Terpestra's c-sample test [26], the llood and Brom c-sample test [22] and Kiefer's K-sample analogues of the Kolmogorov-Smirnov and Cramér-von Mises tests [12] are a few of then. Tests for two-sample problems have been proposed by Wilcoron [29], Mann and Initney [19], Mood and Brown [22], Lehmann [15] and others. Consistency and power properties of some of these tests have been discussed by Lehmann [15], [16], [17], Mood [23], Van Dantzig [5] and others. An exhaustive review of this problem is given in Kruskal and Wallis [14] and Scheffé [25].

The $H$ test of Kruskal and Vallis is a direct generallzation of the two-sample Wilcoxon test discussed in detail by Mann and Witney [19], and its limiting distribution has been derived by Kruskal [13] under the nuIl hypothesis and by Andrews [I] under an alternative hypothesis. These results are generalized by those of the present paper conceming the limiting distribution of the $\mathcal{L}$ test.

The problem discussed in this paper orlginated from the paper of Chernorf and Savage [2] and had its basis in the paper of Hodges and Lehmann [10]. In their paper "The efflciency of some nonparametric competitors of the t-test" [10], Hodges and 

Lehmann discussed the asymptotic efficiency of the Wilcoxon test with respect to all translation alternatives. In the same paper they conjectured that the normal score test whtch was known to be as efficlent as the t-test for normal alternatives [35] is at least as efficient as the t-test for all other alternatives. The validity or this conjecture was established by Chernoff and Savage [2], who developed a new theorem for asymptotic normality of normal score test statistics for the two-sample problem and by a varlational argument proved the Hodges-Lehmann conjecture. The work presented here is an attempt toward generalizing these results to the c-sample problem.

Formally, we may state the c-cample problem as follows. Let $\left[x_{1 j}, j=1, \ldots, m_{1} ; i=1, \ldots, c\right]$ be a set of independent random variables and let $F^{(i)}(x)$ be the probability distribution of $x_{1 j^{\circ}}$ The set of admissible hypotheses designates that each $F^{(1)}(x)$ belongs to some class of distribution functions $\Omega$. The hypothesis to be tested, say $H_{O}$, specifies that $F(1)$ is an element of $\Omega$, for each 1 , and that furthermore

$$
F^{(l)}(x)=\ldots=F^{(c)}(x) \text { for all real } x \text {. }
$$

The class of alternatives to $\mathrm{H}_{0}$ can be considered to be all sets $\left(F^{(1)}(x), \ldots, F^{(c)}(x)\right)$ which belong to $\Omega$ but which violate (2.1). To avold the problem of ties, it is assumed throughout that the class $\Omega$ is the class of continuous distribution functions. 

After finding sufficient conditions for the joint asymptotic normality of $T_{1 i, j} ; j=l, \ldots, c$, we study the limiting dis tributions of a under sequences of admissible alternative hypothesis $H_{n}^{P}$ which specifies that for each $1=1,2, \ldots, c$; $F^{(i)}(x)=F\left(x+\theta_{1} / \sqrt{n}\right)$ with $F \varepsilon \Omega$ but not specified further, and for some pair $(i, j), \theta_{i} \neq \theta_{j}$ where the $\theta_{i}$ 's are real numbers. Limiting probability distributions of $\mathcal{L}$ will then be found as $n \rightarrow \infty$. The problem will be so formulated that $m_{1}(n) / n$ tends to some limit $s_{i}, 0<s_{1}<\infty$, as $n$ tends to $\infty$ 。

3i The proposed test and its relationship to other tests. The over-all sample consists of $\sum_{i=1}^{c} m_{i}=N$ independent random variables $x_{1 j}\left(i=1, \ldots, c ; j=1, \ldots, m_{i}\right)$, where the first subscript refers to the subsample and the second subscript indexes observations within a subsample. Under the null hypothesis all the X's have the same continuous but unknomn c.d.f. (cumulative distribution function) $F(x)$.

Let $Z_{N, 1}^{(j)}=1$, if the $i$ th smallest observation from the combined sample of size $N$ is from the $j$ th sample and otherwise let $z_{N, i}^{(j)}=0$. Denote

$$
m_{j} T_{I N, j}=\sum_{i=1}^{N} z_{N, i}^{(j)} E_{T, i}
$$

where $E_{N, I}$ are given numbers. Then we propose to consider the test statistic $\mathcal{L}$ defined as 

$(3.2)$

$$
d=\sum_{j=1}^{C} n_{j}\left[\left(T_{N, j}-\mu_{H, j}\right) / A_{N}\right]^{2}
$$

where $\mu_{N, j}$ and $A_{N}$ are normalizing constants for the statistics $T_{N, j} ; j=1, \ldots, c$.

The $\mathcal{L}$ test presented in this paper includes as special cases a number of well-knom tests. For example, when $E_{N, 1}=1 / N$, $1 t$ becomes the Kruskal-Wallis $H$ test which is a direct generalization of the two-sample Wilcoxon test and is related to Terpestra's $K$-sample test [26]. When $c=2$ and $E_{N, 1}$ is the expected value of the ith order statistic from the standard normal distribution, then the $\mathcal{L}$ test coincides with the symmetrical two-tail version of the normal score test, also known as the Fisher-Yates-Terry-Hoeffaing $c_{1}$ test and which is asymptotically equivalent to Van der Waerden's test [30], [31]. For it is the: seen that

$$
\mathcal{L}=\frac{N}{N-m_{1}}\left[\sum_{i=1}^{m_{2}} E\left(v^{\left(s_{i}\right)} \mid s_{1}\right)\right]^{2}
$$

where $V^{(1)}<\ldots<V^{(N)}$ is an ordered sample of size If from a standard normal distribution, and $s_{1}<\ldots<s_{m_{2}}$ are the ranks of $x_{21}, \ldots, x_{2 m_{2}}$ from the combined sample. See Lehmann [17], pp. 236-237. When $c=2$, and $E_{N, i}=|1 / 2-1 / N|$, the L-test reduces to the Freund-Ansari test [8] for testing the equality of dispersion of two populations. 

4. Assurntions and notations, Let $x_{11}, \ldots, x_{i m_{1}}$ be the ordered observations of a random sample from a population with continuous cod.i. (cumulative distribution function) $F^{(i)}(x)$; $i=1, \ldots, c$. Let $N=\sum_{i=1}^{c} m_{i}$ and $\lambda_{i}=m_{1} / N$ and assume that for all $N$, the ine gualities $0<\lambda_{0} \leqq \lambda_{1}, \ldots, \lambda_{c} \leq 1-\lambda_{0}<1$ hold for some fixed $\lambda_{0} \leqq 1 / c$.

Let

$$
\left.S_{m_{1}}^{(1)}(x)=m_{1}^{-1} \quad \text { (number of } x_{1 j} \leqq x, j=1, \ldots, m_{i}\right)
$$

be the sample c.dof. of the $m_{i}$ observations in the 1 th set. We shall omit the subscript $m_{i}$ whenever this causes no confusion.

Define

$$
H_{N}(x)=\lambda_{1} S_{m_{1}}^{(1)}(x)+\ldots+\lambda_{c} S_{m_{c}}^{(c)}(x)
$$

Thus $H_{I J}(x)$ is the combined sample c,d.f. The combined population cod.f. is

$$
H(x)=\lambda_{I} F^{(I ;}(x)+\ldots+\lambda_{C} F^{(c)}(x) .
$$

Even though $H(x)$ depends on $N$ through the $\lambda^{\prime} s$, our notation suppresses this fact for convenience and also because our limit theorems are uniform with respect to $F^{(1)}, \ldots, F^{(c)}$ and $\lambda_{1}, \ldots, \lambda_{c} \cdot$ 

Let $z_{N, i}^{(j)}=1$ if the $i$ th smallest of $N=\sum_{i=1}^{c} m_{i}$ observations is from the $j$ th set and otherwise let $z_{N, i}^{(j)}=0$. Denote

$$
(4.1) \quad \tau_{N, j}=m_{j} \cdot T_{N, j}=\sum_{i=I}^{N} E_{N, i} z_{N, j}^{(j)}
$$

where the $E_{\mathrm{N}, 1}$ are Given numbers. Following Chernoff and Savage [2], we shall use the representation

$$
\text { (4.2) } \quad \mathrm{T}_{\mathrm{N}, j}=\int_{-\infty}^{\infty} \mathrm{J}_{\mathrm{N}}\left[\mathrm{H}_{\mathrm{lN}}(x)\right] \mathrm{dS}_{\mathrm{m}_{j}}^{(j)}(x)
$$

where $E_{N, i}=J_{N}(i / N)$. While $J_{N}$ need be defined only at $I / N, 2 / I, \ldots, N / N$, we shall find it convenient to extend its domain of definition to $(0,1]$ by letting $J_{N}$ be constant on $(1 / \mathrm{N},(1+1) / \mathrm{N}]$.

Let

$$
I_{N}=\left[x: 0<I_{N S}(x)<I\right] \text {. }
$$

Then $I_{N}$ is a random interval, given by

$$
I_{N}=\left[x^{(1)}, x^{(N)}\right)
$$

where $\mathrm{X}^{(1)}<\ldots<\mathrm{X}^{(\mathrm{N})}$ denote the $\mathrm{N}$ observations arranged according to size.

Throughout, $K$ will be used as a generic constant which may depend on $J_{i V}$ but will not depend on $F^{(1)}, \ldots, F^{(c)}$, $m_{1}, \ldots, m_{c}$ and $N$. The methods used in the proof for the asymptotic normality of the $T_{N}, j$ 's are mainly adaptations of the methods of Chermorf and Savage [2]. 
'

.

• 
5. Joint asymototic normalíd. Berore proving the asymptotic normality of the $\mathrm{T}_{\mathrm{N}}, \mathrm{j}^{\prime} \mathrm{s}$ we state a fen elementary results.
$(5.1)$
$H \geqq \lambda_{i} F^{(i)} \geqq \lambda_{O} F^{(i)} ; \quad i=1, \ldots, c$.
$(5 \cdot 2)$
$1-F^{(1)} \leqq \frac{1-H}{\lambda_{1}} \leq \frac{1-H}{\lambda_{0}} ; \quad 1=1, \ldots, c 0$
$(5.3)$
$F^{(i)}\left(I-F^{(i)}\right) \leqq \frac{H(1-H)}{\lambda_{i}^{2}} \leqq \frac{H(I-H)}{\lambda_{0}^{2}} ; \quad i=1, \ldots, c$.
$(5.4)$
$d H \geqq \lambda_{i} d F^{(i)} \geq \lambda_{O} d F^{(i)} ; \quad i=1, \ldots, c$.

Lemma 5.2 . If

(1) $J(H)=\lim _{1 \rightarrow \infty} J_{N}(H)$ exists for $O<H<I$ and is not constant,

(2) $\int_{I_{N}}\left[J_{N}\left(H_{N}\right)-J\left(H_{N}\right)\right] d S_{m_{j}}^{(j)}(x)=o_{p}\left(N^{-(1 / 2)}\right)$,

(3) $J_{N}(I)=o(\sqrt{N})$

(4) $\left|J^{(i)}(H(x))\right|=\left|\frac{d^{1} J(H)}{d H^{1}}\right| \leqq K[H(I-H)]^{-i-(I / 2)+\delta}$

for $1=0,1,2$, and for some $\delta>0$, and almost all $\times($ a,$a \cdot x)$,

then, for flyed $F^{(1)}, \ldots, F^{(c)}$ and $\lambda_{1}, \ldots, \lambda_{c}$, 

(5.5) $\lim _{N \rightarrow \infty} P\left(\frac{T_{1, j}-\mu_{H, j}}{\sigma_{N, j}} \leqq t\right)=\int_{-\infty}^{t} \frac{1}{\sqrt{2 \pi}} e^{-x^{2} / 2} d x$,

where

(5.6)

$$
\mu_{N, j}=\int_{-\infty}^{+\infty} J[H(x)] d T(j)(x)
$$

and

(5.7) $N \tau_{N, j}^{2}$

$=2 \sum_{\substack{i=1 \\ 1 \neq j}}^{c} \lambda_{1} \int_{-\infty<x<y<\infty} \int^{(1)}(x)\left[1-F^{(i)}(y)\right] J \cdot[H(x)] J \cdot[H(y)] d F^{(j)}(x) d F^{(j)}(y)$

$+\frac{2}{\lambda_{j}} \sum_{\substack{i=1 \\ i \neq j}}^{c} \lambda_{i}^{2} \iint_{-\infty<x<y<\infty} F^{(j)}(x)\left[1-F^{(j)}(y)\right] J \cdot[H(x)] J \cdot[H(y)] d F^{(i)}(x) d F(i)(y)$

$+\frac{1}{\lambda_{j}} \sum_{\substack{1, k=1 \\ 1 \neq k \\ 1 \neq j \\ k \neq j}}^{c} \lambda_{1} \lambda_{k}\left[\iint_{-\infty<x<y<\infty} F(j)(x)[1-F(j)(y)] J \cdot[H(x)] J \cdot[H(y)] d F(i)(x) d F(k)(y)\right.$

$\left.+\iint_{-\infty<y<x<\infty} F^{(j)}(y)\left[1-F^{(j)}(x)\right] J \cdot[H(x)] J \cdot[H(y)] d F^{(1)}(x) d F^{(k)}(y)\right] \cdot$

\section{Proof.}

$$
\begin{aligned}
T_{N, j} & =\int_{x=-\infty}^{x=+\infty} J_{N}\left[H_{N N}(x)\right] d S_{m_{j}}^{(j)}(x) \\
& =\int_{\left[x: 0<H_{N}(x)<1\right]}\left[J_{N}\left[H_{N}(x)\right]-J\left[H_{N N}(x)\right]\right] d S_{m_{j}}^{(j)}(x) \\
& +\int_{\left[x: 0<H_{N}(x)<1\right]} J\left[H_{N N}(x)\right] d S_{m_{j}}(j)(x)+\int_{\left[x: H_{N}(x)=1\right]} J_{N}\left[H_{N N}(x)\right] d S_{m_{j}}(j)(x) .
\end{aligned}
$$



In the second incegral, wrliing $\operatorname{dS}_{\mathrm{m}_{j}}^{(j)}(x)=\mathrm{d}_{j} \mathrm{~S}_{\mathrm{m}_{j}}^{(j)}(x)-$ $\left.F^{(j)}(x)+F^{(j i}(x)\right)$,

$$
\begin{aligned}
& J\left[H_{\mathrm{N} N}(x)\right]=J\left[H(x !]+\left[H_{\mathrm{IJ}}(x)-H(x)\right] J \cdot[H(x)]\right.
\end{aligned}
$$

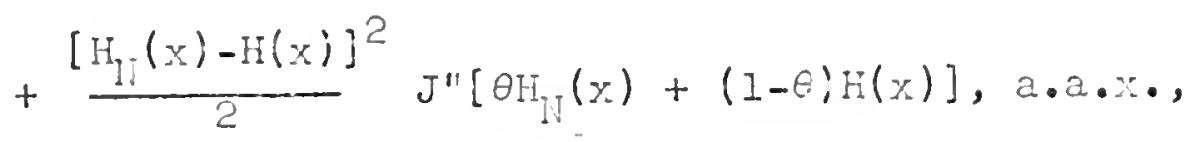

where $0<\theta<I$; and $H(x)=\sum_{i=I}^{C} \lambda_{i}{ }^{(i)}(x)$, and simplifying, we obtain

$$
T_{1 N, j}=A+B_{1 N}+B_{2 N}+\sum_{i=1}^{c+4} C_{i N}
$$

where

$$
\begin{aligned}
& \text { (5.8) } A=\quad J[H(x)] C_{C F}(j)(x) \\
& {[x: 0<\operatorname{II}(x)<I]} \\
& \text { (5.9) } \quad B_{1 N}=\int_{[x: 0<H(x)<I]} J[H(x)] d\left[S_{m_{j}}^{(j)}(x)-F(j)(x)\right] \\
& (5.10) \quad B_{2 N}=\int_{[x: 0<H(x)<I]}\left[H_{N J}(x)-H(x)\right] J \cdot[H(x)] d F(j)(x) \\
& (5.11) \\
& C_{1, N}=\lambda_{1} \int_{[x: 0<H(x)<1]}\left[S_{m_{1}^{(1)}(x)-F}^{(1)}(x)\right] J \cdot[H(x)] d\left[S_{m_{j}}^{(j)}(x)-F{ }^{(j)}(x)\right] \\
& i=1, \ldots, c \text { 。 } \\
& \text { (5.12) } \quad C_{C+1, N}=\int_{I_{I N}} \frac{\left[H_{N}(x)-H(x)\right]^{2}}{2} J^{\prime \prime}\left[\theta H_{N N}+(1-\theta) H\right] d S_{m_{j}}(j)(x) \text {. } \\
& \text { (5.13) } \quad C_{C+2, N}=J_{I_{I !}}\left[J_{N}\left[H_{N}(x)\right]-J\left[H_{N N}(x)\right]\right] d S_{m_{j}}^{(j)}(x) \text {. }
\end{aligned}
$$





$$
\begin{aligned}
& \text { (5.14) } \quad C_{C+j, 11}=\int_{H_{N}=1} J_{N}\left[H_{N}(x)\right] \operatorname{aS}_{\mathrm{m}_{j}}(j)(x) .
\end{aligned}
$$

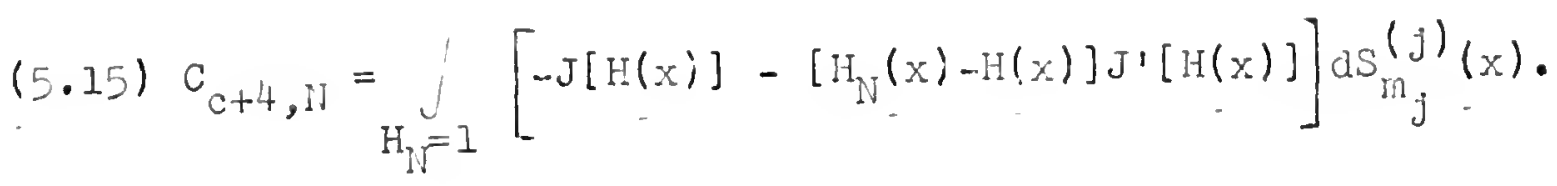

The proof of the lemma is accomplished by showing that ( 1 ) the A-term is nonrandom and finite, (ii) $B_{11}+B_{21 j}$ has a Gaussian distribution in the limit and (iii) the $c$ terms are of higher order.

That the term

$$
A=\int_{[x: 0<H(x)<I]} J[H(x)] d F^{(j)}(x)
$$

is finite and nonrandom follows from assumption 4 of lemrna 5.1; see also in tilis conrection [2], p. 985, and in the appendix we have shown that une $C$ terms are of higher order. Thus, all that is required is to prove,

\section{Sub-lenma 5.I. $B_{1 N}+B_{2 N}$ has a Gaussian distribution in} the limit.

Proof. Integracing $\mathrm{B}_{2 \mathrm{~N}}$ by parts, replacing $\mathrm{H}_{\mathrm{N}}(\mathrm{x})-\mathrm{H}(\mathrm{x})$ by $\sum_{i=1}^{c} \lambda_{1}\left[S_{m_{i}}^{(i)}(x)-F(i)(x)\right]$, and adding $B_{1 N}$ to $i t$, we obtain

$$
\begin{aligned}
(5.16) B_{I N} & +B_{2 N}=-\sum_{\substack{i=1 \\
i \neq j}}^{c} \lambda_{i} \int_{x=-\infty}^{x=+\infty} B(x) d\left[S_{m_{1}}^{(i)}(x)-F(i)(x)\right] \\
& +\int_{x=-\infty}^{x=+\infty}\left[J[H(x)]-\lambda_{j} B(x)\right] d\left[S_{m_{j}}^{(j)}(x)-F(j)(x)\right],
\end{aligned}
$$





$$
\begin{aligned}
(5.17)= & -\sum_{\substack{i \neq 1 \\
i \neq j}}^{u}\left[\lambda_{i} \cdot \frac{1}{m_{i}} \sum_{k=1}^{m_{i}}\left\{B\left(x_{i k}\right)-E B\left(x_{i}\right) j\right]\right. \\
& +\frac{1}{m_{j}} \sum_{k=1}^{m}\left\{J\left[H\left(x_{j k}\right)\right]-\lambda_{j} B\left(x_{j k}\right)-E\left[J\left[H\left(x_{j}\right)\right]-\lambda_{j} B\left(x_{j}\right)\right]\right\}
\end{aligned}
$$

where

$$
B(x)=\int_{x_{0}}^{x} J \cdot[i(y)] d F(j)(y)
$$

With $x_{0}$ determined somewhat arbitrarily, say by $H\left(x_{0}\right)=1 / 2$; $E$ represents the expectation and $x_{1}, \ldots, x_{c}$ have the $F^{(I)}, \ldots, F(c)$ distriblitions respectively.

The c summations given by $(5.17)$ involve independent samples of Identically distributed random variables. Therefore, if we show that the rirst two morients of these random variables exist, then we can apply the central limit theorem, with the result that each sum when properly normalized will have normal distribution in the limit and hence the sum of $c$ summations will have normal distribution in the limit,

First, to turn our attention to moments, note that by assumption 4 of lemma 5.1 and $\mathrm{dF}^{(j)} \leqq\left(1 / \lambda_{0}\right) \mathrm{dH}$,

$$
|B(x)| \leqq K \cdot[H(x)[1-H(x)]]^{-(1 / 2)+\delta}
$$

and proceeding as in [2], for any $\delta^{\prime}$ such that $\left(2+\delta^{\prime}\right)(-1 / 2+6)$ $>-1$

$$
E_{F}(i)[|B(X)|]^{2+\sigma^{\prime}} \leqq K ; \quad i=1, \ldots, J-1, j+1, \ldots, c .
$$


3 
Since

$$
\left|J(H(x))-\lambda_{j} B(x)\right| \leqq K[H(x)(1-H(x))]^{-(1 / 2)+\delta}
$$

the existence of $2+51$ absolute monents of all the terms in equation (5.17) follows.

To compute the variance of $B_{1 N}+B_{2 N}$, note that

$$
\begin{aligned}
& -\lambda i \int_{-\infty}^{+\infty} B(x) d\left[S_{m_{i}}^{(i)}(x)-F^{(i)}(x)\right] \\
& =\lambda_{i} j_{-\infty}^{+\infty}\left[S_{m_{i}}^{(i)}(x)-F^{(1)}(x)\right] J i[H(x)] d F^{(j)}(x), \\
& i=1, \ldots, j-1, j+1, \ldots, c,
\end{aligned}
$$

has mean zero and variance

$$
\begin{aligned}
& \text { (5.19) E\{ }\left\{\lambda_{i} \int_{-\infty}^{+\infty}\left[\mathrm{S}_{i}(i)(x)-\mathrm{F}^{(i)}(\mathrm{x})\right] \mathrm{J}:[\mathrm{H}(\mathrm{x})] \mathrm{dF}(j)(x)\right\}^{2} \\
& =E\left\{\lambda_{i}^{2} j_{-\infty}^{+\infty} j_{-\infty}^{+\infty}\left[S_{m_{i}}^{(i)}(x)-F^{(i)}(x)\right]\left[S_{m_{i}}^{(i)}(y)-F^{(i)}(y)\right]\right. \\
& \left.J^{\ell}[H(x)] J:[H(y)] d F^{(j)}(x) d F^{(j)}(y)\right\} \\
& =\frac{2 \lambda}{N} \int_{-\infty<x<y<\infty} F^{(i)}(x)\left[I-F^{(i)}(y)\right] J^{\prime}[H(x)] J^{\prime}[H(y)] \\
& d F^{(j)}\left(x_{0}\right) d F^{(j)}(y), \\
& i=1, \ldots, j-1, j+1, \ldots, c \text {. }
\end{aligned}
$$



Note that the application of Fubini's theorem permits the interchange or integral and expectation.

By a similar areument, the variance of

$$
\begin{aligned}
& \int_{-\infty}^{+\infty}\left[J(H)(z i)-\lambda_{j} B(x)\right] d\left[S_{m_{j}}^{(j)}(x)-F(j)(x)\right] \\
& \left.=-\sum_{\substack{j=I \\
i \neq j}}^{c} \lambda_{1} \int_{-\infty}^{+\infty}\left[S_{m_{j}}^{(j)}(x)-F(j)(x)\right] J(H j x)\right) d F(i)(x)
\end{aligned}
$$

is given by

$$
\begin{aligned}
& \text { (5.20) } \frac{2}{N \lambda_{j}} \sum_{\substack{i=I \\
i \neq j}}^{c} \lambda_{i}^{2} \int_{-\infty<x<y<\infty} F^{(j)}(x)\left[I-F^{(j)}(y)\right] J\left([H(x)] J^{\prime}[H(y)]\right. \\
& \mathrm{dF}^{(i)}(x) \mathrm{dF}^{(i)}(y) \\
& +\frac{1}{N \lambda_{j}} \sum_{\substack{1, j=1 \\
i \neq k}}^{c} \lambda_{i} \lambda_{k} \int_{-\infty<x<y<\infty} F^{(j)}(x)\left[I-F^{(j)}(y)\right] J(H(x)] J([H(y)] \\
& \begin{array}{l}
1 \neq j \\
k \neq j
\end{array} \\
& d F^{(i)}(x) d F^{(k)}(y) \\
& +\frac{I}{N \lambda_{j}} \sum_{\substack{1, k=1 \\
1 \neq k}}^{C} \lambda_{1} \lambda_{k} \int_{-\infty<y<x<\infty} \int_{<}{ }^{(j)}(y)[I-F(j)(x)] J \cdot[H(x)] J[H(y)] \\
& \begin{array}{l}
i \neq j \\
k \neq j
\end{array} \\
& \mathrm{dF}(i)(x) d F^{(k)}(y)
\end{aligned}
$$

Adding the $c$ terms given by (5.19) and (5.20) we obtain the variance result stated in $(5.7)$.

Thus we have shown that $B_{1 N}+B_{2 N}$ is the sum or $c$ independent terms, each of which has mean zero and finfte absolute $2+\delta^{\prime}$ moments. Hence sub-lemma 5.1 rollows. 
1 
We shall now extend the proos of the above lemma to the case where $F^{(I)}, \ldots, F(c)$ and $\lambda_{I}, \ldots, \lambda_{c}$ are not fixed. We want to find a set of sufficient conditions under wich the asymptotic normality holds unifomly with respect to $F^{(1)}, \ldots, F^{(c)}$ and $\lambda_{1}, \ldots, \lambda_{C}$. For this we need the followine theorem of Esseen [6], p. 430

Theorem (Esseen) 5.1. Let $x_{1}, \ldots, x_{n}$ be independent observations from a population wish mean zero, variance $\sigma^{2}$ and finite absolute $2+\delta^{1}$ moments $\beta_{2+\delta 1,0<\sigma^{1} \leqq}$, then

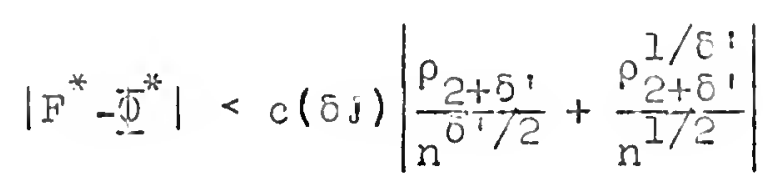

where $F^{*}$ is the cod.f. of $\overline{\mathrm{X}}, \bar{\Phi}^{*}$ is the approximating normal cod.f., $c\left(\delta^{\prime}\right)$ is a finite positive constant only depending on $\sigma^{\prime}$ and $p_{2+\delta^{3}}=\beta_{2+5 i} / \sigma^{2-\delta^{i}}$. (If $\delta^{\prime}=1$, then $\left.\left|F^{*}-\bar{\varphi}^{*}\right|<c\left(\delta^{\prime}\right) \rho_{3} / \sqrt{n}\right)$. To apply this theorem in our situation, it suffices, since we have shom that the $A$ term is finite and the $C$ terms are uniformiy o ${ }_{p}\left(N^{-(I / 2)}\right)$, to prove the uniform convergence of $B_{1 N}+B_{2 N^{\circ}}$ For this it suffices to bound $\rho_{2+\delta^{\prime}}=\beta_{2+\delta^{\prime}} / \sigma^{2+\delta^{\prime}}$ for $B\left(X_{1}\right), \ldots, B\left(X_{C}\right)$. Since in the above lemma we already bounded the absolute $2+\delta^{\prime}$ moments, all that is required is to bound the variances of $B\left(X_{1}\right), \ldots, B\left(X_{c}\right)$ away from zero. Thus we have

\section{Concliany 5.1. If conditions 1 to 4 of lemma 5.1 are} satisfied, and $F(1)$ and $\lambda_{1}, 1=1, \ldots, c$ (where $0<\lambda_{0} \leq \lambda_{1}, \ldots, \lambda_{c} \leqq 1-\lambda_{0}<1$ holds for some flxed 

$\left.\lambda_{0} \leqq 1 / c\right)$ are resericted to a set for which the variances of $B\left(X_{I}\right), \ldots, B\left(X_{c}\right)$ are bounded aray from zero, then the asymptot1c normality holds unirormly with respect to $F(1), \ldots, F(c)$ and $\lambda_{1}, \ldots, \lambda_{c} \cdot$

Next we prove

Lemma 5.2. Under the assumptions of lemma 5.1, the random vector $\sqrt{N}\left(T_{N, j}-\mu_{N, j} ; \ldots ; T_{N, c}-\mu_{N, c}\right)$ has a limitine normal distribution.

Proof. The difference $\sqrt{\mathbb{N}}\left(T_{N, j}-\mu_{N, j}\right)-\sqrt{N}\left(B_{1 N}^{(j)}+B_{2 N}^{(j)}\right)$, where $B_{1 N}^{(j)}+B_{2 N}^{(j)}$ is the "B $B_{I N}+B_{2 N}$ " term for the $j$ th component $T_{\mathrm{IN}, j}-\mu_{\mathrm{NI}, j}$, tends to zero in probability and so, by a well known theorem ([3], p. 299), the vectors $\sqrt{N}\left(T_{N, j}-\mu_{N} ; \ldots ; T_{N, c}-\mu_{N, c}\right)$ and $\sqrt{N}\left(B_{1 N}^{(1)}+B_{2 N}^{(1)} ; \ldots ;\right.$ $\left.B_{1 N}^{(c)}+B_{2 N}^{(c)}\right)$ possess the same limiting distributions. Now since the $j$ th component $B_{1 N}^{(j)}+B_{2 N}^{(j)}$ can be expressed as $\sum_{i=1}^{c}\left\{\frac{1}{m_{1}} \sum_{\alpha=I}^{m_{i}} B_{i j}^{*}\left(x_{1 \alpha}\right)\right\}$, the proof of the lemma follows by applying the Central Limit Theorem to each of the $c$ independent vectors $\frac{1}{m_{1}} \sum_{\alpha=1}^{m_{i}}\left[B_{i 1}^{*}\left(x_{i \alpha}\right), B_{i 2}^{*}\left(x_{i \alpha}\right), \ldots, B_{i c}^{*}\left(x_{i \alpha}\right)\right] ; i=1, \ldots, c$. 6. The Covariance of two B-Statist1cs. By definition $(6.1)$

$$
\begin{aligned}
& \operatorname{Cov}\left(B_{1 N}^{(j)}+B_{2 N}^{(j)}, B_{1 N}^{\left(j^{\prime}\right)}+B_{2 N}^{\left(j^{\prime}\right)}\right)=E\left(B_{1 N}^{(j)}+B_{2 N}^{(j)}\right)\left(B_{1 N}^{\left(j^{\prime}\right)}+B_{2 N}^{\left(j^{\prime}\right)}\right) \\
& =E\left(B_{1 N}^{(j)} B_{2 N}\left(J^{\prime}\right)\right)+E\left(B_{2 N}^{(j)} B_{1 N}^{(j l)}\right)+E\left(B_{2 N}^{(j)} B_{2 N}^{(j ')}\right)
\end{aligned}
$$



where

$$
\begin{aligned}
& (6.2) \quad B_{I N}^{\left(j^{\prime}\right)}=\int_{[x: 0<H(x)<1]} J[H(x)]\left[S_{m^{\prime}}^{\left(j^{\prime}\right)}(x)-F^{\left(j^{\prime}\right)}(x)\right] \\
& (6.3) \quad B_{2 N}^{\left(j^{\prime}\right)}=\int_{[y: 0<H(y)<1]}\left[H_{N}(y)-H(y)\right] J i[H(y)] d F^{\left(j^{\prime}\right)}(y)
\end{aligned}
$$

and $B_{1 j}^{(j)}$ and $B_{2 j}^{(j)}$ are given by $(5.9)$ and $(5.10)$ respectively. Now integrating $B_{I N}^{(j)}$ by parts and using the facts that

$$
\begin{gathered}
\int_{-\infty}^{+\infty} d\left[S_{m_{j}}^{(j)}(x)-F^{(j)}(x)\right]=0 \\
d H(x)=\sum_{i=1}^{c} \lambda_{1} d F^{(i)}(x)
\end{gathered}
$$

and

$$
H_{I T}(y)-H(y)=\sum_{i=1}^{c} \lambda_{r}\left[S_{m_{r}}^{(r)}(y)-F^{(r)}(y)\right]
$$

routine computations yield, for $j \neq j^{\prime}$,

$$
\begin{aligned}
& B_{1 N}^{(j)} B_{2 N}^{\left(j^{\prime}\right.}=-\sum_{i=I}^{c} \frac{c}{\sum_{r=I}} \lambda_{1} \lambda_{r} \int_{x=-\infty y y=-\infty}^{x=+\infty o y=+\infty}\left[\int_{m_{j}}^{(j)}(x)-F^{(j)}(x)\right] \\
& {\left[S_{m_{r}}^{(r)}(y)-F^{(r)}(y)\right] J \cdot[H(x)] J \cdot[H(y)] d F^{(1)}(x) d F^{\left(j^{\prime}\right)}(y) .}
\end{aligned}
$$

Therefore,

$(6.4) E\left(B_{1 N}^{(j)} B_{2 N}^{(j J)}\right)=\frac{I}{N} \sum_{i=I}^{C} \lambda_{I} \iint_{-\infty<x<y<\infty} F(j)(x)[I-F(j)(y)] J \cdot[H(x)] J \cdot[H(y)]$

$$
d F^{(i)}(x) d F^{\left(y^{\prime}\right)}(y) \text {. }
$$



$-\frac{1}{N} \sum_{i=I}^{c} \lambda_{i} \iint_{-\infty<y<x<\infty} F(j)(y)[I-F(j)(x)] J \cdot[H(x)] J \cdot[H(y)]$
$\underset{d F}{(i)}(x) \mathrm{dF}^{(j ')}(y)$.

Proceeding analogously

(6.5) $E\left(B_{2 N} B_{i N}\right)$

$$
\begin{aligned}
& =-\frac{1}{N} \sum_{i=I}^{C} \lambda_{I} \int_{-\infty<x<y<\infty} F^{\left(j^{\prime}\right)}(x)\left[I-F^{\left(j^{\prime}\right)}(y)\right] \cdot J \cdot[H(x)] \\
& \cdot J \cdot[H(y)] d F^{(i)}(x) d F^{(j)}(y) \\
& -\frac{1}{N} \sum_{i=1}^{C} \lambda_{i} \int_{-\infty<y<x<\infty} F^{\left(j^{\prime}\right)}(y)\left[I-F^{\left(j^{\prime}\right)}(x)\right] \cdot J \cdot[H(x)] \\
& \text { - } J \cdot[H(y)] d F^{(1)}(x) d F^{(j)}(y)
\end{aligned}
$$

and

$$
\begin{aligned}
& \text { (6.6) } \quad E\left(B_{2 I I^{\prime N N}} B^{\prime}\right)=\frac{1}{N} \frac{C}{i=1} \lambda_{1} \iint_{-\infty<x<y<\infty} F^{(i)}(x)\left[1-F^{(i)}(y)\right] \\
& \text {. } J \cdot[H(x)] \cdot J \cdot[H(y)] d F^{(j)}(x) d F^{\left(j^{\prime}\right)}(y) \\
& +\frac{1}{N} \sum_{i=1}^{c} \lambda_{1} \quad \iint_{-\infty<y<x<\infty} F^{(i)}(y)\left[1-F^{(1)}(x)\right] \\
& \cdot J \cdot[H(x)] \cdot J^{\prime}[H(y)] d F^{(j)}(x) d F^{(j \prime)}(y) \cdot
\end{aligned}
$$

Thus 


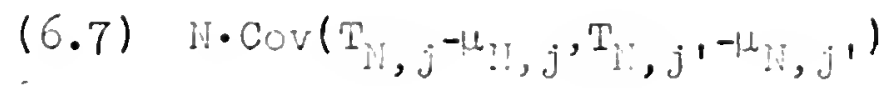

$$
\begin{aligned}
& =-\sum_{i=1}^{c} \lambda_{i}\left[\int_{-\infty<x<y<\infty} F^{(j)}(x)[I-F(j)(y)] \cdot J \cdot[H(x)]\right. \\
& \cdot J \cdot[H(y)] d F^{(i)}(x) d F^{\left(J^{\prime}\right)}(y) \\
& +\int_{-\infty<y<\cdots<\infty} F^{(j)}(y)\left[1-F^{(j)}(x)\right] \cdot J \cdot[H(x)] \cdot J \cdot[H(y)] \\
& \left.\cdot d F^{(i)}(x) a F^{\left(j^{\prime}\right)}(y)\right] \\
& -\sum_{i=I}^{c} \lambda_{i} \int_{-\infty<x<y-\infty} F^{\left(j^{\prime}\right)}(x)\left[I-F^{(j \prime)}(y)\right] \cdot J \cdot[H(x)] \cdot J \cdot[H(y)] \\
& \cdot d F^{(i)}(x) d F^{(j)}(y) \\
& +\int_{-\infty<y<x<\infty} F^{\left(j^{\prime}\right)}(y)\left[I-F^{\left(j^{\prime}\right)}(x)\right] \cdot J^{\prime}[H(x)] \cdot J^{\prime}[H(y)] \\
& \text { - } \left.\mathrm{dF}^{(i)}(x) \mathrm{dF}^{(j)}(y)\right] \\
& +\sum_{i=I}^{c} \lambda_{i}\left[\sum_{-\infty<x<y<\infty} F^{(i)}(x)[]-F^{(i)}(y)\right] \cdot J \cdot[H(x)] \cdot J '[H(y)] \\
& \cdot d^{(j)}(x) d F^{\left(j^{\prime}\right)}(y) \\
& +\underbrace{}_{-\infty<y<x<\infty} F^{(i)}(y)[I-F(i)(x)] \cdot J \cdot[H(x)] \cdot J \cdot[H(y)] \\
& \left.\cdot d F^{(j)}(x) d F^{\left(j^{\prime}\right)}(y)\right], j \neq j^{\prime} \cdot
\end{aligned}
$$

Combining the metrial of the previous two sections produces 

Theorem 6.1. Under the assumptions of lemma 5.1, the random vector $T=\left(\sqrt{N}\left(T_{N, I}-\mu_{N, I}\right), \ldots, \sqrt{N}\left(T_{N, c}-\mu_{N, c}\right)\right)$ has a liniting normal distribution with zero mean vector and_variance - covariances given by limiting forms of (5.7) and $(6.7)$ respectively as $N \rightarrow \infty$.

Remark. The following theorem gives a simple sufficient condition under which conditions 1,2 , and 3 of lemma 5.1 hold.

Theorem 6.2. If $J_{N}(i / N)$ is the expectation of the ith orier statistic of a sample of size $N$ from a population whose cumulative distribution function is the inverse function of $\mathrm{J}$ $\mid J^{(1)}[H(x)] \leqq K[H(I-H)]^{-i-(I / 2)+\delta}$ for $i=0,1,2$; for some $\delta>0$ and a.a. $x$, then 

(1) $\lim _{H \rightarrow \infty} J_{N H}(\mathrm{H})=J(H)$.

(ii) $J_{I !}(I)=O(\sqrt{N})$.

(i11) $\int_{I_{N}}\left[J_{N}\left(H_{N}\right)-J\left(H_{N}\right)\right] d S_{m_{j}}^{(j)}(x)=o\left(N^{-(I / 2)}\right) ; j=1, \ldots, c$.

Remark 1. The condition $\left|J^{(i)}[H(x)]\right| \leqq K[H(I-H)]^{-i-(I / 2)+\delta}$ a.a. $x$ is weaker than the condition $\left|J^{(i)}(H)\right| \leqq$ $K[H(I-H)]^{-i-(I / 2) \div \delta}$ usea by Chemorf and Savage [2], otherwise theorem 6.2 is the generalization of the latter's theorem 2 .

Remark 2. With the use of this theorem, it is easy to verify that if $J_{N}(i / N)$ is the expected value of the ith order statistic of a sample of size if from (i) the standard nomal distribution, (ii) the logistic distribution, (1ii) the double exponential distribution, (iv) the exponential distribution, then the vector $\left(T_{i, I} ; \ldots ; T_{N, c}\right)$ has a limiting normal distributæon.

Ii The limiting distribution of \& underpitmanis shift alternatives. From this section onward, we assume that $m_{1}, \ldots, m_{c}$ are nonciecreasing functions of a natural number $n$ that tends to infinity. The dependence on $\mathrm{n}$ is indicated when necessary, by uriting $m_{i}(n), \mu_{N, i}(n)$, etc. For convenience, it is assumed that, for all i,

$$
\lim _{n \rightarrow \infty} \frac{m_{1}(n)}{n}=s_{i}
$$



exists, and there exist tro constants a and b such that $0<a<s_{1}<b<\infty$.

In subsequent analysis, we shall concern ourselves with a sequence of admissible alternative hypothesis $\mathrm{H}_{\mathrm{H}}^{\mathrm{P}}$ which specifies that for each $1=1, \ldots, c ; F^{(1)}(x)=F\left(x+\theta_{1} / \sqrt{n}\right)$ uith $f \varepsilon \Omega$ but not speciried further, and for some pair $(i, j)$, $\theta_{1} \neq \theta_{j}$. The letter $n$ is used to index a sequence of situations in which $\mathrm{H}_{\mathrm{n}}^{\mathrm{P}}$ is the true hypothesis. Limiting probability distribution of $\mathcal{L}$ will then be found as $n \rightarrow \infty$.

We first prove the following

Theorem 7.1. If

(1) for all i,

$$
\lim _{n \rightarrow \infty} \frac{m_{i}(n)}{n}=s_{i}
$$

exists,

(2) conditions (1) to (4) of lemma 5.1 are satisfied,

(3)

$$
F(j)(x)=F\left(x+\theta_{j} / \sqrt{n}\right)
$$

so that for each index $n$, the hypotheses $H_{n}^{P}$ are valici, then the random vector $\left[\sqrt{m_{I}}\left(T_{N, I}-\mu_{N, I}\right) \ldots\right.$, $\left.\sqrt{m_{c}}\left(T_{N, c} \mu_{N, c}\right)\right]$ has a limiting normal distribution witr zero means and covariance matrix whose $\left(j, j^{\prime}\right)$ th term is

$(7.1)$

$$
\left(\delta_{j j^{\prime}}-\frac{\sqrt{s_{j} j^{\prime}}}{\sum_{i=I}^{c} s_{i}} A^{2}\right.
$$

Where 
$(7 \cdot 2)$

$$
A^{2}=\int_{0}^{1} J^{2}(x) d x-\left(\int_{0}^{1} J(x) d x\right)^{2}
$$

\section{and the Innit holds uniformly in $s_{i}$ provicied}

$0<a<s_{i}<b<\infty ; i=1, \ldots, c$.

Proof. From equation $(5.7)$

(7.3) $\lim _{n \rightarrow \infty} N \cdot c_{1}^{2}, j=\left[\sum_{\substack{i=I \\ i \neq j}}^{c} s_{i}+\frac{I}{s_{j}} \sum_{\substack{i=1 \\ i \neq j}}^{c} s_{i}^{2}+\frac{I}{2 s_{j}} \sum_{\substack{i, k=1 \\ i \neq k \\ i \neq j \\ k \neq j}}^{c} s_{i} s_{k}\right] I_{I} / \sum_{r=1}^{c} s_{r}$

$$
+\frac{1}{2 s}\left(\sum_{\substack{i, k=1 \\ i \neq k \\ i \neq j \\ k \neq j}}^{c} s_{i} s_{k}\right) I_{2} / \sum_{r=I}^{c} s_{r}
$$

Where

$$
\begin{aligned}
(7.4) \quad I_{1} & =2 \underset{0<x^{<y<1}}{\int} x(I-y) J^{\prime}(x) J^{\prime}(y) 2 x d y, \\
(7.5) & =\int_{0}^{1} J^{2}(x) d x-\left(\int_{0}^{1} J(x) d x\right)^{2}
\end{aligned}
$$

and

$$
\begin{aligned}
& \text { (7.6) } I_{2}=2 \int_{0<y<x<1} \mathrm{~J}(I-x) \mathrm{J}(x) \mathrm{J}^{\prime}(y) d x d y \text {, } \\
& (7.7) \\
& =\int_{0}^{1} J^{2}(x) d x-\left(\int_{0}^{1} J(x) d x\right)^{2} .
\end{aligned}
$$

Thus, omitting the routine algebra,

$$
\lim _{n \rightarrow \infty} N \cdot \frac{2}{N, j}=\left(\begin{array}{c}
\frac{c}{i=1} \\
-1+\underbrace{s}_{j}
\end{array}\right) A^{2}
$$



Similarly, rrom equation (6.7),

$$
\lim _{n \rightarrow \infty} N \operatorname{Cov}\left(T_{N, j}-\mu_{N, J}: T_{I T, j},-\mu_{I I, j \mu}\right)=-A^{2} .
$$

Hence using Tleorem 6.1, we obtain the desired result.

Denoting $\sqrt{m}_{j}\left(T_{N}, j^{-\mu}, j\right) / A$ by $\|_{j}$, it now follows that the random vector $:=\left(w_{l}, \ldots, w_{c}\right)$ has a limiting normal distribution with zero mean vector and with covariance matriz whose $(j, j ')$ th tern is

$$
\left(\delta_{j, j}-\frac{\sqrt{s_{j} j^{\prime}}}{\sum_{i=1}^{c} s_{i}}\right)
$$

We now make the analysis of variance cransformation

$$
\begin{aligned}
& s_{0}=\sum_{i=1}^{c} \sqrt{e_{i}}, H_{1} \text {, where } e_{i},=\sum_{i=1}^{s_{i}} s_{1} \\
& S_{1}=\sum_{i^{\prime}=1}^{c} a_{i i}, W_{i}, ; i=1,2, \ldots, c-1
\end{aligned}
$$

where the a's are chosen to make ine transformation orthogonal. It follows that $\sum_{i=I}^{c} w_{i}^{2}$ is asymptotically chi-square with $c-1$ degrees of freedom.

Now recalling that

$$
W_{i}=\sqrt{m_{i}}\left[T_{N, i}-\mu_{N, i}(\theta)\right] / \Lambda
$$


.. 
and letting

$$
r_{i}=\sqrt{m_{i}}\left[\mu_{H, i}(\theta)-\mu_{H, i}(0)\right] / A
$$

we write $\mathcal{L}$ as

$$
\alpha=\sum_{i=I}^{c}\left(w_{i}+r_{i}\right)^{2}
$$

and this has the same limiting distribution as

$$
\dot{L}^{*}=\sum_{i=1}^{c}\left(w_{i}+r_{i}^{*} i^{2}\right.
$$

where $r_{i}^{*}=\lim _{n \rightarrow \infty} r_{i}$ reduces to

$$
r_{i}^{*}=\lim _{n \rightarrow \infty} \sqrt{m_{1}}\left[J _ { - \infty } ^ { + \infty } \left[J\left\{\frac{c}{\sum_{\alpha=I} \lambda} F\left(x+\frac{\theta_{\alpha}-\theta_{1}}{\sqrt{n}}\right]-J\{F(x)\} \mid d F(x)\right] / A .\right.\right.
$$

We assume that the above limit exists and is finite. Noting that $\sum_{i=I}^{c} \sqrt{s_{i}}=0$ and $\sum_{i=I}^{c} \sqrt{s_{i}} r_{i}^{*}=0$, it follows from a theorem of Mann and Vald [20] that

Theorem 7.$)$ Suppose that for all $i, \underset{n \rightarrow \infty}{\lim } m_{1} / n=s_{i}$ exists and is positive. Then under the hypothesis $\mathrm{H}_{\mathrm{n}}^{\mathrm{P}}$, if for any real numbers $t_{1}, \ldots, t_{c}$,

$$
\left.\lim _{n \rightarrow \infty} \sqrt{m_{1}}\left[\int_{-\infty}^{+\infty}\left[J \sum_{\{i=1}^{c} \lambda_{1} F\left(x+\frac{t_{i}}{\sqrt{n}}\right)\right\}-J\{F\{x)\}\right] d F(x)\right] / A
$$



exists and is finite, then for $\mathrm{n} \rightarrow \infty$, the Iimitin distribution of the statistic $\mathcal{L}$ is $x_{c-1}^{2}\left(\lambda^{L}\left(F_{n}^{P}\right)\right)$ where $\lambda^{L}\left(H_{n}^{P}\right)$ is the noncentral1ty parameter iven by

$$
\text { (7.8) } \begin{aligned}
\lambda^{L}\left(H_{n}^{P}\right)=\sum_{j=I}^{c}\left[\operatorname { l i m } _ { n \rightarrow \infty } \sqrt { m _ { j } } \int _ { - \infty } ^ { + \infty } \left[J \left\{\frac{c}{\sum_{\alpha=I} \lambda} \alpha^{F}\left(x+\frac{\theta_{\alpha}-\theta}{\sqrt{n}}\right\}\right.\right.\right. \\
-J\{F(x)\}] d r(x)]^{2} / A^{2} .
\end{aligned}
$$

Remark. If the function $J$ is such that $J(u)=u$, then from (7.8), letting $m_{j}=n \cdot s_{j}$, we obtain for $\lambda{ }^{\mathcal{L}}\left(H_{n}^{P}\right)$ the expression

$$
\frac{12}{\left(\sum_{i=1}^{c} S_{1}\right)^{2}} \sum_{j=1}^{c} s\left(\frac{c}{\sum_{\alpha=1}^{s}} \lim _{n \rightarrow \infty} \int_{x=-\infty}^{x=+\infty} \sqrt{n}\left\{F\left(x+\frac{\theta^{-\theta} j}{\sqrt{n}}\right)-F(x)\right\} d F(x)\right)^{\prime}
$$

which is the noncentrallty parameter $\lambda^{\mathrm{H}}\left(\mathrm{H}_{n}^{\mathrm{P}}\right)$ of the Kruskalfallis $H$ test. (See Andrews [1], p. 726).

In many situations, the noncentrality parameter $\lambda^{\mathcal{L}}$ can be computed easily with the aid of the following lema which, though stated in a form appropriate to our purpose, is due to Hodges and Lehmann [II].

\section{Lemma 7.2 (Hodses-Lehmann). If}

(1) F is a continuous cumulative distribution function, differentiable in each of the open intervals $\left(-\infty, a_{1}\right),\left(a_{1}, a_{2}\right)$, $\ldots,\left(a_{s-1}, a_{s}\right),\left(a_{s}, \infty\right)$ and the derivative of $F$ is bounded in each of these intervals and 

(ii) the function $\frac{d}{d x} J[F(x)]$ is bounded as $x \rightarrow \pm \infty$

tinen

$$
\begin{aligned}
& \text { (7.9) } \lim _{n \rightarrow \infty} \sqrt{n} J_{-\infty}^{+\infty}\left[J\left\{\sum_{\alpha=1} \lambda_{\alpha} F\left(x+\frac{\theta_{\alpha}-\theta}{\sqrt{n}} j\right)\right\}-J\{F(x)\}\right] d F(x) \\
& =\frac{I}{\sum_{i=I}^{c} S_{i}} \sum_{\alpha=I}^{c} \alpha_{a}\left(\theta_{\alpha}-\theta_{j}\right) j_{-\infty}^{+\infty} \frac{d}{d x} J\{F(x)\} d F(x) .
\end{aligned}
$$

The proof of this lemma follows by the methods used in section 3 and 4 or Hodiges-Lehmann (1961).

In case the conditions of lenma 7.2 are satisfied, then

(7.10) $\quad \lambda^{\alpha}\left(H_{n}^{p}\right)=\sum_{\alpha=1}^{c} S_{\alpha}\left(\theta_{\alpha}-\bar{\theta}\right)^{2}\left(j_{-\infty}^{+\infty} \frac{d}{d x} J[F(x)] f(x) d x\right)^{2} / A^{2}$

where

$(7.11)$

$$
\bar{\theta}=\sum_{\alpha=1}^{c} s_{\alpha}^{\theta} \alpha / \sum_{\alpha=1}^{e} s_{\alpha}
$$

and $A^{2}$ is defined in $(7 \cdot 2)$.

8. Asymptotic relative efficiency. The concept of asymptotic relative efficiency of one test with respect to another is due to Pitman. An exposition of his work, together with some extensions is presented by lioether [27].

Theorem 8.1. If $m_{i}=n \cdot s_{1}$, and is the distribution function $F$ is such that

(1) $\lim _{n \rightarrow \infty} \sqrt{n} \int_{-\infty}^{+\infty}\left[F\left(x+\frac{\theta}{\sqrt{n}}\right)-F(x)\right] d F(x)$ exists 


It may be remarked that (8. $)$ agrees vith the results found by Chernoff-Savage [2] and Hodges-Lehmann [11] for the two-sample case, and hence the resultis of this paper as vell as those of [2] apply directly to the c-sample problem.

The asymptotic relative efficiency of the classical fost Wth respect to an arbitrary $\mathcal{L}$ test is contained in the following

Theorem 8.2. If

(i) forall i, $\lim _{n \rightarrow \infty} \frac{m_{i}(n)}{n}=s_{i}$ exists and is positive,

(ii) the distribution function $F(x)$ satisfies the assumptions of lemma $7 \cdot 2$, and

(iii) $\int_{-\infty}^{+\infty} x^{2} \mathrm{dF}(x)-\left(\int_{-\infty}^{+\infty} \mathrm{ydF}(x)\right)^{2}=\sigma^{2}$ exists,

then, the asymptotic relative efficiency of the classical f test with respect to an arbitrary $\&$ test for testing the hypothesis $\mathrm{H}_{0}$ against $\mathrm{H}_{\mathrm{n}}^{\mathrm{P}}$ is

(8.3)

$$
e_{f_{1}, \mathcal{L}}^{P}(F(x))=\frac{A^{2}}{\sigma^{2}}\left(\frac{1}{\int_{-\infty}^{+\infty} \frac{d}{d x} J[F(x)] d F(x)}\right)^{2}
$$

Proof. The Fistatistic is defined as

$$
Z_{1}=\frac{\frac{1}{c-I} \sum_{i=1}^{c} m_{i}\left(x_{i}-\bar{X}\right)^{2}}{\frac{1}{N-c} \sum_{i=1}^{c} \sum_{j=1}^{m_{i}}\left(x_{i j}-x_{i}\right)^{2}}
$$



where $x_{i} .=\sum_{j=I}^{m_{i}} x_{i j} / m_{i}$ and $\bar{x}=\sum_{i=I}^{c} \sum_{j=I}^{m_{i}} x_{i j} /$ N. It has been shown by Andrevs [1] that under the hypothesis $H_{n}^{P}$, this has a limiting noncentral chi-square distribution with $c-1$ degrees of freedom and noncentrality parameter $\lambda^{F^{7}}\left(H_{n}^{P}\right)$ given by $(8.5)$

$$
\lambda^{F}\left(H_{n}^{P}\right)=\sum_{i=1}^{c} s_{1}\left[\left(\theta_{1}-\bar{\sigma}\right) / \sigma\right]^{2}
$$

Now proceeding by standard arguments, the proof follows. In particular, when $J=\Phi$, where $\Phi$ is the standard cumulative nomal distribution function having the density $\phi$, $(8.6)$

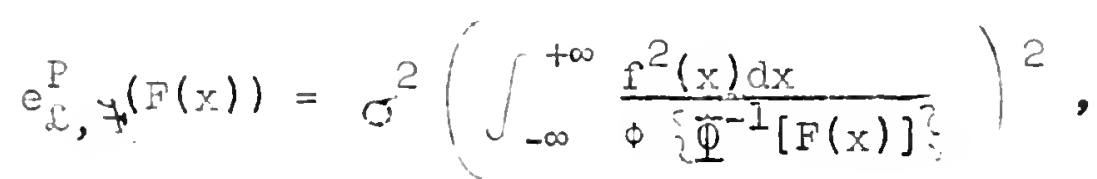

Which is known to be the asymptotic efficiency of the two sample normal scores test with respect to the student's t-test and is always 1 . When $F(x)$ is normal distribution function, this is 1. See In this connection Chernoff-Savage [2] and Hodges-Lehmann [1]].

2. Acknowledsment. I wish to express my sincere thanks to Professor Erich L. Lehman for proposing this investigation and for the generous help and guidance during its progress. 

APPEIDIX

10. Higher orier terms. Before we prove that the $\mathrm{C}$ terms of lemma 5.1 are uniformly of higher order, we state the following elementary results which are used repeatedly. (Also in this connection see Chernorf and Savase [a].)

10A. EIementary Presilts.

1. $H \geqq \lambda_{1} F^{(1)} \geqq \lambda_{0} F^{(i)}$; $i=1, \ldots, c$.

2. $1-F^{(1)} \leqq \frac{1-H}{\lambda_{1}} \leqq \frac{1-H}{\lambda_{0}} ;$ $1=1, \ldots, c$.

3. $F^{(1)}(1-F(i)) \leqq \frac{H(1-H i}{\lambda_{1}^{2}} \leqq \frac{H(I-H)}{\lambda_{0}^{2}} ; \quad i=1, \ldots, c$.

4. $\quad \mathrm{dH} \geqq \lambda_{1} d F(i) \geqq \lambda_{O} d F^{(1)}$ $1=1, \ldots, c$.

5. Let $\left(a_{N I}, b_{I I}\right)$ be the interval $S_{11}$ where

$(10.1)$

$$
S_{V_{\varepsilon}}=\left[X: H(1-H)>\eta_{\varepsilon} \lambda_{O} / N\right],
$$

when $\eta_{\varepsilon}$ can be chosen independent of $F^{(i)}$ and $\lambda_{i} ; i=1, \ldots, c$, such that

(10.2) $\quad P\left[X_{1 j} \in S_{1 !} ; i=1, \ldots, c ; j=1, \ldots, m_{i}\right] \geqq 1-\varepsilon$.

10B. Detalied consideration of the c-terms of lemma 5.1 .

First, let us consider 

$(10.3)$

$$
\begin{aligned}
C_{1 N} & =\lambda_{i} \int_{0<H<1}\left[S_{m_{i}}^{(i)}(x)-F^{(i)}(x)\right] J^{\prime}[H(x)] d\left[S_{m_{j}}^{(j)}(x)-F^{(j)}(x)\right] ; \\
& i=1, \ldots, j-1, j+1, \ldots, c \\
& =\lambda_{1}\left[C_{l i}^{(i)}+C_{2 N}^{(i)}\right] ; \quad i=1, \ldots, c ; i \neq j,
\end{aligned}
$$

where

$(10.4)$

$$
\begin{gathered}
C_{I N}^{(i)}=\int_{S_{N_{E}}}\left[S_{m_{i}}^{(i)}(x)-F^{(i)}(x)\right] J^{\prime}[H(x)] a\left[S_{m_{j}}^{(j)}(x)-F^{(j)}(x)\right] \\
i=1, \ldots, c ; i \neq j,
\end{gathered}
$$

and

$(10.5)$

$$
C_{2 N}^{(i)}=\int_{S_{N \in}}\left[S_{m_{i}}^{(i)}(x)-F^{(i)}(x)\right] J^{\prime}[H(x)] d\left[S_{m_{j}}^{(j)}\langle(x)-F(j)(x)] ;\right.
$$

$$
1=1, \ldots, c ; i \neq j
$$

First note that

$(10.6)$

$$
E\left(C_{I N}^{(i)}\right)=E\left[E\left(C_{I N}^{(i)} \mid x_{j 1}, \ldots, x_{j m_{j}}\right)\right]=0 ; \quad 1=1, \ldots, c ; 1 \neq j \cdot
$$


!

: 
Next

$$
\begin{aligned}
& {\left[C_{1 N}^{(1)}\right]^{2}=2 \int_{\substack{x, y \in S_{N} \\
x<y}}\left[S_{n_{E}}^{(1)}(x)-F^{(1)}(x)\right]\left[S_{m_{i}}^{(i)}(y)-F^{(i)}(y)\right] J^{\prime}[H(x)] J^{\prime}[H(y)]} \\
& \left.d\left[S_{m_{j}}^{(j)}(x)-F^{(j)}(x)\right] d{ }_{j}^{j} S_{m_{j}}^{(j)}(y)-F^{(j)}(y)\right] \\
& +\frac{1}{m_{j}} \int_{x \in S_{N}}\left[S_{m_{i}}^{(i)}(x)-F^{(i)}(x)\right]^{2}\left[J^{\prime}[H(x)]\right]^{2} d S_{m}(j)(x) ; \\
& i=I, \ldots, c ; i \neq j \text {. }
\end{aligned}
$$

Therefore,

(10.7) $E\left(C_{I N}^{(i)}\right)^{2}=E\left[E\left[\left(C_{I N}^{(i)}\right)^{2} \mid x_{j l}, \ldots, x_{j m}\right]\right]$

$$
\begin{aligned}
& -\frac{2}{m_{i} m_{j}} \int_{\substack{x, y \in S_{N} \\
x<y}} F(1)(y)\left[I-F^{(i)}(y)\right] J^{\prime}[H(x)] J^{\prime}[H(y)] \\
& d F^{(j)}(x) d F^{(j)}(y) \\
& +\frac{1}{m_{i}^{m} j} \int_{x \in S_{I J}} F^{(1)}(x)\left[1-F^{(I)}(x)\right]\left[J^{\prime}[H(x)]\right]^{2} d F^{(j)}(x) ;
\end{aligned}
$$

$$
i=1, \ldots, c ; i \neq j
$$

$\leq \frac{K}{N^{2}} \int_{x, y \in S_{N_{\varepsilon}}} H(x)[1-H(y)][H(x)(1-H(x))]^{-3 / 2+6}[H(y)(1-H(y))]^{-3 / 2-t}$

$$
+\frac{K}{N^{2}} \int_{x \in S_{N}} H(x)[I-H(x)]\left[H(x)(I-H(x)]^{-3+26} d H(x) d H(y)\right.
$$$$
\Leftrightarrow \frac{K}{N^{2}}+\frac{K \eta_{\varepsilon}^{-1: 26}}{N^{1+2 \delta}}=o\left(\frac{1}{i j}\right) ; \quad[K \text { is generic }] .
$$ 

Hence from $(10.6)$ and $(10.7)$, we obtain, us ne the larkofi inequality, $(10.8)$

$$
\left|C_{111}^{(1)}\right|=o_{p}\left(N^{-(1 / 2)}\right) 。
$$

We no: consider $C_{21 J}^{(1)}$. Let $H_{1}=H\left(a_{N}\right), H_{2}=H\left(b_{H}\right)$. Then from (10.1) $\mathrm{H}_{1}=\mathrm{I}-\mathrm{H}_{2}<\mathrm{K} / \mathrm{N}$. With probability greater than $1-\varepsilon$, there are no observations in $\overline{\mathrm{S}}_{\mathrm{H}_{\varepsilon}}$ and

$(10.9)$

$$
\begin{aligned}
& \left|C_{2 N}^{(1)}\right| \leqq \int_{0}^{H} I F(i)(x)\left[J^{\prime}[H(x)]\right] d F(j)(x) \\
& +\int_{\mathrm{H}_{2}}^{1}\left(1-\mathrm{F}^{(1)}(\mathrm{x})\left[J^{\prime}[\mathrm{H}(\mathrm{x})]\right] d \mathrm{~F}^{(j)}(\mathrm{x})\right. \\
& i=1, \ldots, c ; i \neq j
\end{aligned}
$$

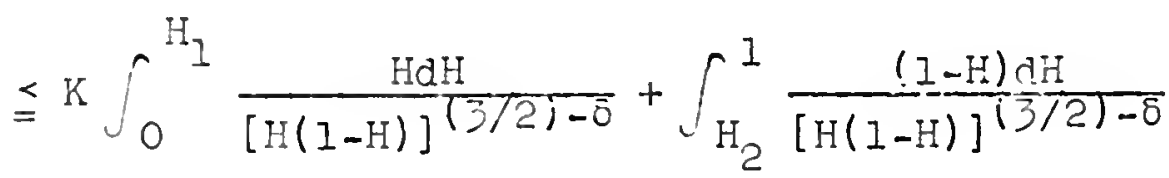

$$
\begin{aligned}
& \leqq K \int_{0}^{H_{1}} H^{-(1 / 2)+\delta} d H \leqq K \frac{I}{N(I / 2)+\delta} .
\end{aligned}
$$

Hence

$(10.10)$

$$
\left|C_{2 N}^{(1)}\right|=o_{p}\left(N^{-(I / 2)}\right) ;
$$$$
i=1, \ldots, c ; i \neq j \text {. }
$$

Consequently,

(10.11) $\quad c_{1 N}=\lambda_{1}\left[c_{1 N}^{(1)}+C_{2 N}^{(1)}\right]=o_{p}(N-(1 / 2)) ; 1=1, \ldots, c ; 1 \neq j$. The proof of $C_{J N}=o_{p}\left(N^{-(I / 2)}\right)$ follows first showing that

$$
c_{j N}=-\frac{\lambda_{j}}{2}\left[c_{11 N}+c_{12 N}-c_{13 N}\right]
$$



where

$$
\begin{aligned}
& \text { (a) } \quad C_{I I N}=\int_{S_{N !}}\left[S_{m}^{(j)}(x)-F^{(j)}(x)\right]^{2} J^{\prime \prime}[H(x)] d H(x) \text {, } \\
& \text { (b) } \quad C_{12 I !}=\int_{S_{1}}\left[S_{m_{j}}^{(j)}(x)-F(j)(x)\right]^{2} J "[H(x)] d H(x) \text {, } \\
& \text { (c) } \quad C_{13 N}=\frac{I}{m_{j}} \int J^{\prime}[H(x)] d S_{n_{j}}^{(j)}(x)
\end{aligned}
$$

and then showing that each $C_{1 K N}$ is $o_{p}(1-(1 / 2)) ; k=1,2,3$. The proofs of the above statement are onitted since they are essentially contained in the work of Chernoff and Savage [2]. Next consider

$$
\begin{aligned}
& C_{C+1, N}=\int_{I_{i N}}\left[H_{N}(x)-H(x)\right]^{2} J^{\prime \prime}\left[\theta H_{N}(x)+(1-\theta) H(x)\right] d S_{m_{j}}^{(j)}(x), \\
& 0<\theta<1 \text {. }
\end{aligned}
$$

With probability $>I-\varepsilon$, the interval $I_{N}$ can be replaced by $S_{N_{\varepsilon}}$ Without changing $C_{C+I, N}$. Furthermore since

$$
\sup _{H_{N}>0}\left|\frac{H(x)}{H_{I f}(x)}\right|=O_{p}(1)
$$

and

$$
\sup _{H_{N}<I}\left|\frac{I-H(x)}{I-H_{i J}(x)}\right|=O_{p}(I),
$$

for each $\varepsilon>0$, there exists an $\eta_{\varepsilon}^{*}>0$ such that with greater 
,

$\checkmark$

. 
than $1-\varepsilon$, we have for $\left\{x: 0<H_{y}(x)<1\right\}$,

$$
\left[\theta H_{N}(x)+(1-\theta) H(x)\right]\left[I-\left\{\theta H_{N}(x)+(1-\theta) H(x)\right\}\right]>\eta_{\varepsilon}^{*} H(x)[1-H(x)] \text {. }
$$

Then

$$
\left|C_{C+1, N}\right| \leqq\left(\eta_{\varepsilon}^{*}\right)-(5 / 2)+\sigma C_{a N}
$$

where

$$
C_{a N}=\int_{S_{N_{\varepsilon}}}\left[H_{N}(x)-H(x)\right]^{2}\{H(x)[1-H(x)]\}^{-(5 / 2)+\delta} \mathrm{dS}_{m_{j}}(j)(x)
$$

and

$$
\begin{aligned}
& E\left(C_{\alpha N}\right)=E\left[E\left(C_{\alpha N} \mid x_{j I}, \ldots, x_{j m_{j}}\right)\right] \\
& =\frac{1}{N} \int_{S_{N}} \sum_{i=1}^{c} \lambda_{i} F^{(i)}(1-F(i))[H(1-H)]^{-(5 / 2)+\delta} d F^{(j)}(x) \\
& +\frac{-}{N^{2}} \int_{S_{N^{\prime}}}\left(1-F^{(j)}\right)\left(1-2 F^{(j)}\right)[H(I-H)]^{-15 / 2)+\delta_{d F}(j)}(x) \\
& \leqq \frac{K}{N} \int_{S_{N_{\varepsilon}}}[H(1-H)]^{-(3 / 2)+\delta} d H+\frac{K}{N^{2}} \int_{S_{N_{\varepsilon}}}[H(1-H)]^{-(5 / 2)+\delta} d H \\
& \leqq \frac{K}{N(1) 2]+\delta} \text {. }
\end{aligned}
$$

Consequently $\quad c_{C+1, N}=o_{p}\left(N^{-(1 / 2)}\right)$.

The negligibility of $\mathrm{C}_{\mathrm{C}+2, N}$ and $\mathrm{C}_{\mathrm{C}+3, \mathrm{~N}}$ follows from assumptions 2 and 3 of lemma 5.1 and the proof of the negligibility of $\mathrm{C}_{\mathrm{C}+4_{4}, \mathrm{~N}}$ proceeds in the same manner as given by Chernoff and Savage for the term $C_{4 N}$ and therefore is not given here. 



\section{REFRENCES}

1. ANDREWS, F. C. (1954). Asymptotic behavior of some rank tests for analysis of variance. Ann. Math. Statist. $25724-735$.

2. CHERNOFF, H. and SAVACE, I. R. (1958). Asymptotic nomality and efficiency of certain non-parametric test statistics. Ann. Math. Statist. $22972-924$.

3. CRAMÉR, Harald (1946). Mathematical Metiods of Statistics. Princeton University Press.

4. CRAMÉR, Harald (1937). Random Variables and Probability Distributions. Cambridge University Press.

5. DANTZIG, D. Van (1951). On the consistency and the power of Wilcoxon's two-sample test. Nederl. Akad. Wetensch.

\section{Proc. 54}

6. ESSEEN, C. G. (1945). Fourier analysis of distribution functions. Acta Math. 27 1-125.

7. FRASER, D. A. S. (1956). Non-parametric Methods of Statistics. John Wiley and Sons, Inc.

8. FREUND, J. E. and ANSARI, A. R. (1957). Two-way rank sum for variances. Virginia Polytechnic Institute.

9. HANNAN, E. J. (1949). The asymptotic powers of certain tests based on multiple correlations. J.Roy. Statist. Soc. B. $2121-219$.

10. HODGES, J. L. JR. and LEHMANN, E. I. (1956). The efficiency of some non-parametric competitors of the t-test. Ann. Math. Statist. 27 324-335. 

11. HODGEs, J. L. JT. and Lamian, E. L. (1,61). Comparison of the normal scores and Wilcoxon tests. Proceedings of the Fourth Borice] ev Sympostum on Wathematical Statistics and Probab11ity. $\frac{1}{\sim}$ 307-317.

12. KIEFER, J. (1959). Kusample analogues of the Kolmogorov Smirnov and Cramer v. Mises tests. Enn Hath. Statist. 20 $420-447$.

13. KRUSKAL, W. H. (1952). A non-parametric test for the several sample problems. Ann. Math. Statist. 23 525-540.

14. KRUSKAL, W. H. and WALLIS, W. A. (1952). Use of ranks in one criterion analysis of variance. J. Amer. Statist. Assoc, $47 \quad 583-621$.

15. Lehmann, E. I. (1951). Consistency and unbiasedness of certain non-parametric tests. Ann. Math. Statist. 22 165-179.

16. LeHninn, E. L. (1953). The power of rank tests. Ann. Math. Statist. 24 23-43.

17. Lemmani, E. L. (1959). Testing Statistical Hypotheses. John Wiley and Sons, Inc.

18. LOEVE, M. (1955). Probab1lity Theory. D. Van Nostrand Co., Inc.

19. MANN, H. B. and WHITNEY, D. R. (1947). On a test of whether one of two random variables is stochastically larger than the other. Ann. Math. Statist. 18 50-60.

20. MANN, H. B. and WALD, A. (1943). On stochastic limit and order relationship. Ann. Math._Statist. 14 217-226. 



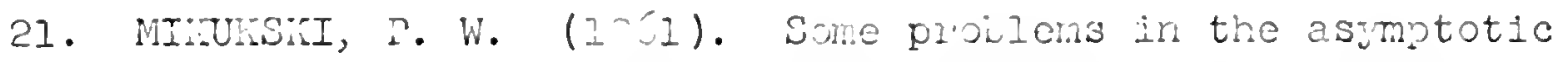
theory of testing statistical hypotheses. Efriciency of non-parametric prccedures. Ph.D. Thesis, University of California.

22. MOOD, A. M. (1950). Introduction to Theory of Statistics. McGraw-Hill Publishing Co.

23. MOOD, A.M. (1954). On the asymptotic efriciency of certain non parametric two-sample tests. Ann. Math. Statist. $25 \quad 514-522$.

24. RAO, C. R. (1952). Advanced Statistical Methods in Biometric Research. John Wiley and Sons, Inc.

25. SCHEFFÉ, H. (1943). Statistical inference in the nonparametric case. Ann. Math. Statist. 14 305-332. 26. TERPESTRA, T. J. (1952). A non-parametric k-sample test and its connection with the H-test. Report S(92) (VP2) of the Statistical Department of the Mathematical Centre, Amsterdam.

27. TERRY, M. E. (1952). Some rank order tests which are most powerful against specific alternatives. Ann. Math. Statist. $23 \quad 346 \cdot 366$.

28. WALD, A. and WOLFOWITZ, J. (1944). Statistical tests based on permutations of the observations. Ann. Math. Statist. $\frac{15}{2} 358-372$.

29. WILCOXON, F. (1245). Individual comparison by ranking methods. Biometrics Bull. I 80-83. 



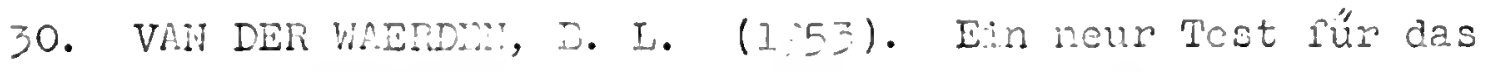
problem der Zwei Stich problem. Math. Annalen. 근 $93-107$.

31. VAN DER WAERDEN, B. I. (1956). The computation of the $\mathrm{X}$ distribution. Proceedings of the rinind Berkeley Symposium on Mathematical Statistics and Drobability. $\therefore 207-203$.

32. Noether, G. E. (1954). On a theorem of Pitman. Ann. Math. Statist. $25 \quad 514-522$.

33. ANSARI, A. R. and BRADLEY, R. A. (1960). Rank sum tests for dispersions. Ann. Math. Statist. 21 1174-1184. 34. SAVAGE, I. R. (1952). Contributions to the theory of rank order statistics: Two-sample case. Ann. Math. Statist. $23 \quad 346-366$.

35. HOEFFDING, W. (1550). Optimum non-parametric tests. Proceedins of the Second Berkeley Symposium on Mathemat1cal_Statistics and_Probability. 83-92. 


$$
\therefore
$$


Approved Distribution List for Unclassified sechnical wejorts

Hear, Statistics Branch

Office or diaval Research

Hashin:ton 25, I. C.

Commanding officer

Offic of taval .esearch

irancir ofice, uavy 100

Fleet post orice

New Yor:., New York

ASTIa Docurent wervice Center

arlint on inll tation

Arlin.ton 12, Virginia

Technical Information Oificer iNaval . .esearch Laboratory

Washiniton 25, D.C.

Institute for Deiense Analyses
Commuications liesearch Division von leumann $\mathrm{Hall}$

Princeton, New Jersey

Bureau of Jupplies and iccounts Code OW

Departient oi the liavy

Washington 25, 2. C.

Professor T.W. Anclerson

Departrent of a tirenatical

Statistics

Columbia University

New Yor's 27, Liew York

Professor $\angle$. . Birnbaum

Laboratory of utatistical

Research

Departivent of iatheilatics

University or iashineton

Seattle 5, haslington

Professor . Dowler
Applied lilatiematics and

Statistical Lab.

Stanford inivorsity

Stanford, Califoria

Professor ialoh A. Jradley

Departient of stat. and

Statistical Lab.

Virginia iolytecinic Institute

Blacksburs, Vircinia
(6) Inst. oI 落th. Sciences

Wen ior: vinvers ty

ITew York 3, New York

(1)

(1) Department of Statistics

-ic icair state University

wast Lainsine, .ichican

Propessor ...rinan Chornorf

aplier iatl. and Stat. Lab.

stariford University

Stanford, California

Professor W.G. Cociran

Iepartinent of Scatictics

iarvard U:iversity

Frofessor Ben.jarnn Lipstein

Dr. iaul G. Hoel

De ratuent of tatientics

ULVES - ty oi California

Los hareles 24, Calizornia

Irofessor arold .otellins

ssociate Director

Institute of tatistics

Univors ty of ort'r Carolnna

Crazel rill, Jorth Carolina

Profes or L. mricz

Sciool o- usiness

sdrinistration

University of Uinnesota

inmeapolis, innesota

Professor Oscar iemitione

statistics iasoratory

(1) Iowa itate Colle:e

slies, Iowa

Dr. Carl F Kossack

I. Ji. Corp. Jata Processing

2601 iain st.

$\therefore$ iouston 2, Texas 

Professor Gerald J.Lieberman

Applied hathematics and

Statistics Laboratory

Stanford university

Stanford, California

Professor William G. Viadow

St.rford ras rach hitute

Menlo Park, California

Professor J. Ileyman

Department of Statistics

University of California

Berkeley 4, California

Professor Herbert Robbins

Mathenatical statistics Jepartment

Columbia. University

New York 27, Iew York

Professor Murray iosentlatt

Department of linthematics

Brown University

Providence, R.I.

PrOFESE. L. J.

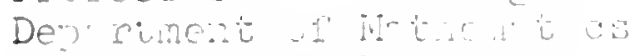

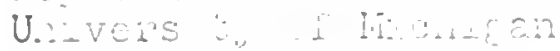

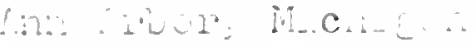

Dr. Herbert Solomon

Department of Statistics

Stanford University

Stanford, California

(1)

Professor Frank Spitzer

Department of ilatiematics

Cornell University

Ithaca, iver York

Professor S.S. iilks

Departmont of liathomatics

Princeton University

Princeton, New Jersey

Professor Evan J, lilliliams

Institute of Statistics

State Collere Soction

North Carolina State Collere

Raleigh, North Carolina

Professor J. Wolfowitz

Dopartment of liatiomatics

Cornell University

Ithaca, ilew Yorls

Professor M.H. iruskal.

Department of Statistics

The University of Chicago

Chicago 37 , Illinois 


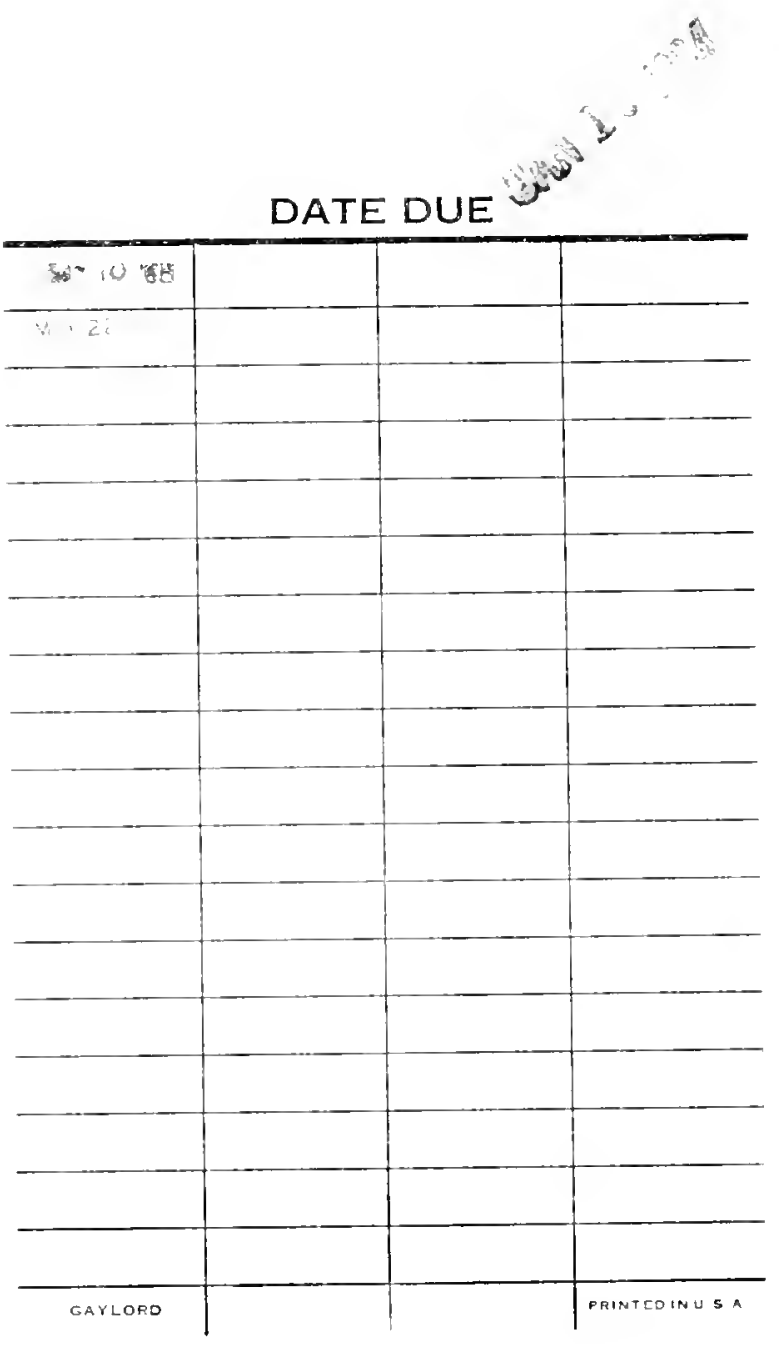




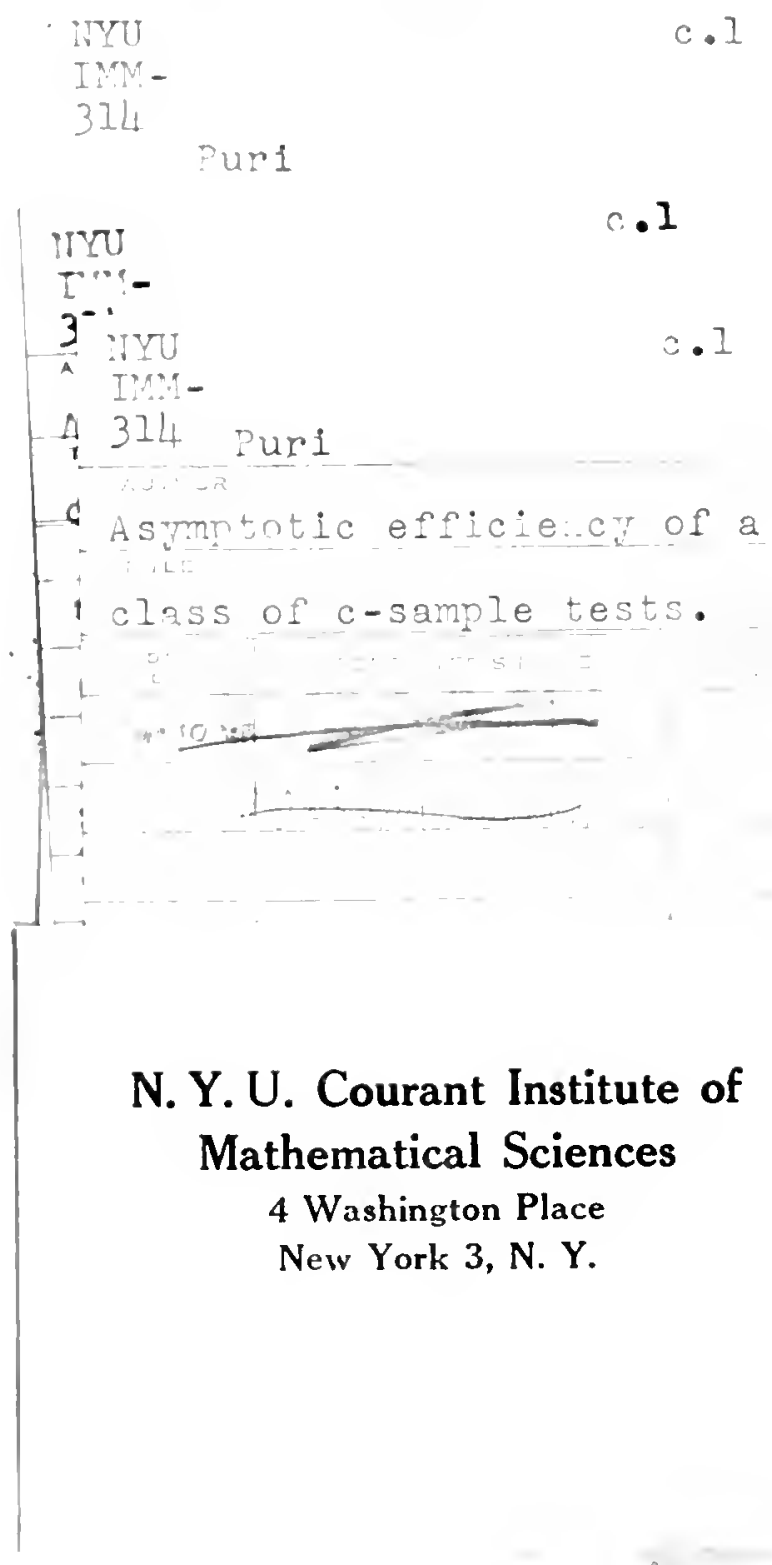


